\title{
Orally administered cladribine displays efficacy in multiple sclerosis trial
}

According to the results of a phase III clinical trial published in the New England Journal of Medicine, orally administered cladribine-an immunomodulatory drug that targets $\mathrm{T}$ and $\mathrm{B}$ lymphocytes-shows efficacy in patients with relapsingremitting multiple sclerosis (MS). "Oral cladribine reduces relapses relative to placebo by $55-58 \%$, and has an impact on disability progression and all MRI outcome markers," states lead investigator Gavin Giovannoni, of Barts and The London School of Medicine and Dentistry, UK. Moreover, according to Giovannoni, cladribine compares favorably with existing MS therapies. "It appears as if cladribine is about twice as effective as the current injectable first-line diseasemodifying drugs," he claims.

\section{Oral cladribine reduces relapses relative to placebo by $55-58 \% \ldots 77$}

Various therapies that either suppress or modulate the immune system have been licensed for relapsing-remitting MS, which is characterized by inflammation, demyelination and axonal degeneration. These agents are only effective in subsets of patients, however, and can be associated with serious adverse effects. Moreover, currently available MS therapies need to be administered parenterally, which can affect patient compliance with medication regimens. Cladribine is one of several potential oral MS drugs in development that, it is hoped, might overcome some of the problems associated with existing agents.

Giovannoni and colleagues randomly assigned 1,326 patients with relapsingremitting MS to orally receive placebo, or cladribine at one of two cumulative doses ( $3.5 \mathrm{mg}$ or $5.25 \mathrm{mg}$ per kg of body weight). The study drugs were administered over a 96 week period in several short courses, which corresponded to 8-20 days of oncedaily treatment per year. The primary end point of the trial was the rate of relapse at 96 weeks, which was measured using the Expanded Disability Status Scale. Various clinical and MRI secondary end points were also assessed.

In comparison with individuals in the placebo group, patients who received cladribine had a reduced annualized relapse rate at 96 weeks ( 0.33 in the placebo group versus 0.14 and 0.15 in the $3.5 \mathrm{mg}$ and $5.25 \mathrm{mg}$ cladribine groups, respectively). Cladribine treatment also led to a number of favorable outcomes on secondary clinical measures of efficacy. More patients treated with cladribine remained relapse free at 96 weeks (79.7\% for the low-dose group and $78.9 \%$ for the high-dose group) than did individuals who received placebo (60.9\%). Furthermore, cladribine treatment was associated with an increase in the time to first relapse. Finally, patients who received cladribine, in comparison with placebo, exhibited a relative reduction in the risk of sustained progression of disability over a period of 3 months (33\% in the low-dose group versus $31 \%$ in the high-dose group).

On brain MRI, both cladribine groups exhibited lower lesion activity at 96 weeks than did individuals in the placebo group, as indicated by the presence of fewer gadolinium-enhancing T1-weighted lesions, active T2-weighted lesions and combined unique lesions.

Several adverse events were associated with cladribine treatment. Notably, the occurrence of lympocytopenia was increased in patients receiving this drug (21.6\% in the low-dose group and 31.5\% in the high-dose group) compared with individuals who received placebo (1.8\%). In addition, 8 patients in the $3.5 \mathrm{mg}$ treatment group and 12 individuals who received the higher dose of cladribine developed herpes zoster, compared with no patients in the control group.

As detailed in their report, the investigators believe that the study showed cladribine to be beneficial in patients with relapsing-remitting MS. Nevertheless, they stress the need to weigh up the treatment benefits with the associated risks.

\section{Darran Yates}

Orignial article Giovannoni, G. et al. A placebo-controlled trial of oral cladribine for relapsing multiple sclerosis. N. Engl. J. Med. 362, 416-426 (2010) 